\title{
Esophageal mucosal integrity improves after laparoscopic antireflux surgery in children with gastroesophageal reflux disease
}

\author{
Femke A. Mauritz ${ }^{1,6}$ - Nicolaas F. Rinsma ${ }^{3}$ - Ernest L. W. van Heurn ${ }^{4}$ - Cornelius E. J. Sloots ${ }^{5}$. \\ Peter D. Siersema $^{6} \cdot$ Roderick H. J. Houwen $^{2} \cdot$ David C. van der Zee $^{1} \cdot$ Ad A. M. Masclee $^{3}$. \\ José M. Conchillo ${ }^{3}$ Maud Y. A. Van Herwaarden-Lindeboom ${ }^{1}$
}

Received: 9 February 2016/ Accepted: 14 October 2016/Published online: 1 November 2016

(c) The Author(s) 2016. This article is published with open access at Springerlink.com

\begin{abstract}
Background Esophageal intraluminal baseline impedance reflects the conductivity of the esophageal mucosa and may be an instrument for in vivo evaluation of mucosal integrity in children with gastroesophageal reflux disease (GERD). Laparoscopic antireflux surgery (LARS) is a well-established treatment option for children with proton pump inhibitory (PPI) therapy resistant GERD. The effect of LARS in children on baseline impedance has not been studied in detail. The aim of this study was to evaluate the effect of LARS on baseline impedance in children with GERD.
\end{abstract}

Femke A. Mauritz and Nicolaas F. Rinsma have contributed equally to this work.

Femke A. Mauritz

f.a.mauritz@umcutrecht.nl

1 Department of Pediatric Surgery, Wilhelmina Children's Hospital, University Medical Center Utrecht, Room: KE.04.140.5, PO Box 85090, 3508 AB Utrecht, The Netherlands

2 Department of Pediatric Gastroenterology and Hepatology, Wilhelmina Children's Hospital, University Medical Center Utrecht, Utrecht, The Netherlands

3 Department of Gastroenterology and Hepatology, Maastricht University Medical Center, Maastricht, The Netherlands

4 Department of Pediatric Surgery, Maastricht University Medical Center, Maastricht, The Netherlands

5 Department of Pediatric Surgery, Erasmus MC - Sophia Children's Hospital, Rotterdam, The Netherlands

6 Department of Gastroenterology and Hepatology, University Medical Center Utrecht, Utrecht, The Netherlands
Methods This is a prospective, multicenter, nationwide cohort study (Dutch national trial registry: NTR2934) including 25 patients [ 12 males, median age 6 (range 2-18) years] with PPI-resistant GERD scheduled to undergo LARS. Twenty-four hour multichannel intraluminal impedance $\mathrm{pH}$ monitoring (MII-pH monitoring) was performed before and 3 months after LARS. Baseline impedance was evaluated during consecutive 2 - $h$ intervals in the 24-h tracings.

Results LARS reduced acid exposure time from $8.5 \%$ $(6.0-16.2 \%)$ to $0.8 \%(0.2-2.8 \%), p<0.001$. Distal baseline impedance increased after LARS from $2445 \Omega$ $(1147-3277 \Omega)$ to $3792 \Omega(3087-4700 \Omega), p<0.001$. Preoperative baseline impedance strongly correlated with acid exposure time $(r-0.76, p<0.001)$; however, no association between symptomatic outcome and baseline impedance was identified.

Conclusions LARS significantly increased baseline impedance likely reflecting recovery of mucosal integrity. As the change in baseline impedance was not associated with the clinical outcome of LARS, other factors besides mucosal integrity may contribute to symptom perception in children with GERD.

Keywords Gastroesophageal reflux disease - Laparoscopic fundoplication $\cdot$ Baseline impedance $\cdot$ Pediatrics

$\begin{array}{ll}\text { Abbreviations } \\ \text { GER } & \text { Gastroesophageal reflux } \\ \text { GERD } & \text { Gastroesophageal reflux disease } \\ \text { GSQ } & \text { Gastroesophageal Reflux Symptom } \\ & \text { Questionnaire } \\ \text { LARS } & \text { Laparoscopic antireflux surgery } \\ \text { MII } & \text { Multichannel intraluminal impedance } \\ \text { PPI } & \text { Proton pump inhibitor }\end{array}$

Abbreviations

GERD Gastroesophageal reflux disease

GSQ Gastroesophageal Reflux Symptom

Questionnaire

LARS Laparoscopic antireflux surgery

PPI Proton pump inhibitor 
Multichannel intraluminal impedance (MII) combined with $\mathrm{pH}$ monitoring is a well-established technique for the assessment of gastroesophageal reflux disease (GERD) in both children and adults [1-4]. Changes in conductivity between multiple electrode pairs on a single-sensor MIIcatheter allow detection of intra-esophageal movement of saliva during swallowing and the occurrence of gastroesophageal reflux (GER). In the absence of GER or swallowing, the esophagus is collapsed and the esophageal wall comes directly in contact with the MII-pH sensor catheter [5]. The impedance value during these periods, commonly referred to as baseline impedance, reflects the intrinsic electrical conductivity of the esophageal wall and may offer an in vivo tool to assess the integrity of the esophageal mucosa [6]. Baseline impedance values in healthy volunteers are usually high, whereas GERD patients express low baseline impedance values. Low baseline impedance values have been associated with Barrett's esophagus or severe esophagitis [7], but recently they were also linked to acid-induced mucosal changes in patients with non-erosive reflux disease $[6,8,9]$.

Furthermore, comparison with the golden standard, the Ussing Chamber technique showed that baseline impedance was closely correlated to TEER and fluorescein permeability as measured with the Ussing chamber [10].

Recovery of impaired mucosal integrity, reflected by increased baseline impedance, may relieve symptoms in GERD patients and be a marker for the clinical outcome of therapy $[6,11]$. Previous studies evaluating the effect of proton pump inhibitors (PPIs) on baseline impedance in children and adults with GERD showed an increase in baseline impedance during acid suppressive therapy [11-13]. However, these studies did not reveal a correlation between the recovery of baseline impedance and symptomatic outcome [12, 13].

Laparoscopic antireflux surgery (LARS) to treat severe, PPI-therapy resistant GERD aims to reduce reflux episodes and symptoms [14]. At present, the effect of LARS on esophageal mucosal integrity has not previously been studied in detail. The aim of this study is to assess the effect of LARS on baseline impedance and to explore if changes in baseline impedance are associated with the clinical outcome of LARS.

\section{Methods}

\section{Study design}

We performed a prospective multicenter study in three University Medical Centers in the Netherlands that are specialized in performing fundoplication in children [Wilhelmina children's Hospital, University Medical
Center Utrecht (UMCU); Sophia's Children's Hospital, Erasmus University Medical Center (Erasmus MC) and Maastricht University Medical Center (MUMC)]. From July 2011 until December 2013, we prospectively included pediatric patients diagnosed with PPI-therapy resistant GERD. All patients had been treated with high dosages of PPI for at least 6 months, and GERD was defined as: (1) troublesome GERD symptoms, (2) pathological reflux on 24-h pH monitoring and (3) a positive SAP (symptom association probability) assessment ( $\geq 95 \%)$.

Patients who underwent previous esophageal or gastric surgery (except previous gastrostomy placement) and those with structural abnormalities other than esophageal hiatal hernia were excluded. Patients were studied before and 3 months after the surgical procedure.

\section{Surgical procedure}

All laparoscopic fundoplication procedures were performed by pediatric surgeons experienced in minimal invasive pediatric surgery. In the UMCU, the anterior, partial fundoplication according to Thal [15] was used to perform fundoplication. The other two centers (Erasmus MC and MUMC) used the posterior, total fundoplication according to Nissen [16]. Before fundoplication, the distal esophagus was fully mobilized, and the distal $3 \mathrm{~cm}$ of the esophagus was repositioned back into the abdomen. Both vagal nerves were identified, and a crusplasty was performed routinely (UMCU and EMC). Thereafter, the fundoplication was constructed. The Thal fundoplication was performed by plicating the fundus of the stomach over $270^{\circ}$ against the distal anterior intra-abdominal part of the esophagus and the diaphragmatic crus [17]. A floppy Nissen was constructed with one of the sutures of the $360^{\circ}$ posterior wrap incorporated in the esophageal wall [16].

\section{Ambulatory 24-h multichannel intraluminal impedance $\mathrm{pH}$ monitoring}

Ambulatory 24-h MII-pH testing was conducted after at least 3-day cessation of all medications that affect gastrointestinal motility and/or acid secretion. Measurements were performed using a combined $\mathrm{pH}$-impedance catheter assembly that consisted of six impedance segments and one ISFET pH electrode (Unisensor AG, Attikon, Switzerland). The $\mathrm{pH}$ electrode was positioned $5 \mathrm{~cm}$ above the upper border of the manometrically localized lower esophageal sphincter (LES). Impedance and $\mathrm{pH}$ signals were stored on a digital data logger (Ohmega, Medical Measurement Systems, Enschede, The Netherlands), using a sampling frequency of $50 \mathrm{~Hz}$. Patients and/or their parents were instructed to continue their regular diet, to report GERD symptoms and to keep a diary of their consumptions and body position (supine or upright) during the measurement. 


\section{Reflux-specific questionnaires}

To assess reflux symptoms, patients and/or their parents were asked to fill out the validated age-adjusted Gastroesophageal Reflux Symptom Questionnaire (GSQ) before and 3 months after LARS [18]. Reflux symptoms and dysphagia were scored for frequency and severity on a score ranging from 1 (none) to 7 (most severe). Symptoms were defined as: no symptoms (no symptoms reported); mild (mild symptoms, weekly); moderate (mild symptoms, daily or severe symptoms, weekly) and severe (severe symptoms, daily). Reflux symptoms were scored using the symptoms heartburn, regurgitation, food refusal and vomiting.

\section{Data analysis}

Baseline impedance values were calculated for two specific segments in the esophagus during consecutive 2-h intervals in the 24-MII-pH tracings as previously described [11]. Periods of $\geq 30$ s not containing any swallows or gastroesophageal reflux episodes were selected, and the averaged impedance value over this specific time period at two of the in total six impedance segments (Z6-distal and Z2-proximal) was calculated, using a specific function incorporated in the analysis software (Ohmega, MMS, Enschede, The Netherlands). The 2-hourly obtained baseline impedance values for each segment were averaged and used for further analysis. The 24-h MII-pH tracings were further analyzed for acid exposure time, the number and acidity of reflux episodes according to previously described definitions [19]. Acid exposure time was defined as pathological when $\mathrm{pH}$ $<4$ during $>6.0 \%$ of time during 24-h monitoring [20, 21]. Reflux episodes reaching the proximal (z2) impedance segment were classified as proximal. Baseline impedance throughout the manuscript refers to baseline impedance in the distal (z6) segment, unless stated otherwise.

\section{Ethical approval and trial registration}

This study was registered with the Dutch national trial registry (www.trialregister.nl; Identifier: 2934). Ethical approval for this prospective multicenter study was obtained from the University Medical Center Utrecht Ethics Committee, and local approval was obtained by the two participating centers (Erasmus MC and MUMC). Prior to initiating any trial-related study procedure, informed consent from the patients' parents was obtained.

\section{Statistical analysis}

Continuous parametric variables were expressed as mean \pm standard error of the mean (SEM). Nonparametric variables were expressed as median, with interquartile ranges (IQR). For continuous parametric outcomes, a paired sample $T$ test was performed. Nonparametric continuous outcomes were analyzed using the Wilcoxon signed-rank test. Correlations between different parameters were calculated using the Pearson correlation coefficient or Spearman's rank correlation coefficient as appropriate. Linear regression analysis was performed to identify possible determinants of the effect of LARS on baseline impedance. Determinants of interest included: age, type of fundoplication and changes in acid exposure time. A $p$ value below 0.05 was considered statistically significant. All statistical analyses were performed using commercially available computer software (IBM SPSS Statistics for Windows, Version 22. Armonk, NY: IBM Corp.).

\section{Results}

In total, 25 children were included in our study. Mean age of the included patients was 6 (range 2-18) years at the time of fundoplication. Five children $(80 \%)$ had normal neurodevelopment $(\mathrm{NN})$, while impaired neurodevelopment (NI) was seen in five children (20\%). Patient demographics are shown in Table 1. Thal fundoplication was performed in 18 and Nissen fundoplication in seven children. In all patients, fundoplication could be completed by laparoscopy.

The caregivers of all 25 patients filled out both pre- and postoperative reflux symptom questionnaires. Pre-operative 24-h pH-MII tracings were completed in all 25 patients. After surgery, 24-h pH-MII could not be performed in two patients due to refusal of the tracing by the caregivers. Postoperative tracings were successfully completed in 23 of the 25 children. Preoperative esophagogastroduodenoscopy was performed in 18 patients. In 13 of these patients, macroscopic and/or microscopic acid-induced changes in the esophagus were observed.

\section{Baseline impedance before antireflux surgery}

Median baseline impedance before surgery was $2245 \Omega$, with a range from 430 to $4401 \Omega$. Spearman's rho correlation coefficient showed a strong negative correlation

Table 1 Baseline characteristics

\begin{tabular}{lc}
\hline & (Median; IQR) \\
\hline Age at time of operation (years) & $6.0(3.0-11.0)$ \\
Duration of hospital admission (days) & $3.0(2.0-4.5)$ \\
\hline & $n(\%)$ \\
\hline Male gender & $12(48.0 \%)$ \\
Impaired neurodevelopment & $5(20.0 \%)$ \\
Gastrostomy preoperatively in situ & $4(16.0 \%)$ \\
\hline
\end{tabular}


between distal baseline impedance and acid exposure time ( $r-0.76, p<0.001)$ before surgery (Fig. 1A). Baseline impedance also negatively correlated with other reflux parameters, such as reflux episodes lasting longer than 5 min $(r-0.55, p=0.005)$ and number of acid reflux episodes $(r-0.55, p=0.005)$. The number of non-acid reflux episodes showed no correlation with baseline impedance $(r-0.03$, NS).

In patients who underwent preoperative endoscopy, a trend toward significance was observed when comparing baseline impedance in patients with macroscopic and/or microscopic signs of esophagitis $(n=13)$ to those with no sign of inflammation $(n=5)$ [resp. 1788 (IQR 677-3187 $\Omega$ ) versus $2928 \Omega \quad(\mathrm{IQR} \quad 2591-4364 \Omega)$, $p=0.09]$. Baseline impedance showed no correlation with severity of reflux symptoms [severe symptoms $2376 \Omega$ (IQR 1251-3275 $\Omega$ ) versus mild/moderate symptoms $2925 \Omega$ (IQR 1083-3207 $\Omega$ ), NS].

Proximal baseline impedance was significantly higher when compared to distal baseline impedance [3116 $\Omega(\mathrm{IQR}$ 2539-4071 $\Omega$ ) versus $2445 \Omega$ (IQR 1147-3277 $\Omega$ ), $p<0.05]$. Baseline impedance in the proximal segment also showed a negative correlation with acid exposure time ( $r-0.62, p=0.001$; Fig. 1B) and number of acid reflux episodes $(r-0.50, p=0.01)$.

Proximal extension of reflux episodes occurred on average in $44.8 \%$ (SEM $4.8 \%$ ) of all reflux episodes. The number of reflux episodes reaching the proximal esophagus did not correlate with proximal baseline impedance $(r-0.18, \mathrm{NS})$.

\section{Effects of laparoscopic antireflux surgery}

LARS reduced acid exposure time from $8.5 \%$ $(6.0-16.2 \%)$ to $0.8 \%(0.2-2.8 \%), p<0.001$, at 3-month follow-up. All other reflux parameters, including the number of proximal and non-acid GER episodes on 24-h MII-pH monitoring, were also significantly reduced after LARS (Table 2). LARS significantly increased baseline impedance in both the distal and proximal impedance segment (Fig. 2). Distal baseline impedance showed a significant correlation with remaining postoperative acid exposure time $(r-0.67, p<0.001)$ (Fig. 3). Furthermore, the change in distal baseline impedance after LARS correlated to the reduction in acid exposure time $(r 0.48$, $p=0.02)$.

The median preoperative baseline impedance was $2445 \Omega$. After LARS, the baseline impedance value in three patients was lower than this median preoperative baseline impedance value (Fig. 3). In two of these three patients, persisting pathologic acid exposure was found. The third patient with lower postoperative baseline impedance after the procedure had esophageal gastric metaplasia in the distal esophagus. Despite normalization of acid exposure, low baseline impedance persisted only in the most distal segment of the esophagus likely due to increased conductivity of gastric epithelium.

Overall reflux symptoms significantly decreased after LARS $(p=0.001)$. In $15(65 \%)$ patients, complete remission of reflux symptoms was achieved and in an additional $6(26 \%)$ patients symptom scores improved. In two of the 23 (9\%) patients, reflux symptoms were unaltered; one of these patients showed a low baseline value $(536 \Omega)$. Deterioration of reflux symptoms did not occur (Table 3). Four patients with postoperative reflux complaints and/or pathological reflux on 24-h $\mathrm{pH}$ monitoring still required PPI therapy.

Similar to preoperative analysis, no association between postoperative symptoms and baseline impedance could be identified [no symptoms $(n=15)$ versus persistent symptoms $(n=8): 3917 \Omega($ IQR 3087-4490) versus $3706 \Omega$ (2983-4373), NS].

\section{Determinants of the effect of LARS on baseline impedance}

A linear regression analysis was performed to explore determinants of the effect of LARS on baseline impedance.
Fig. 1 Correlation between esophageal acid exposure time (\%) and $\mathbf{A}$ distal baseline impedance $(\Omega)$ and $\mathbf{B}$ proximal baseline impedance $(\Omega)$ in GERD patients before LARS
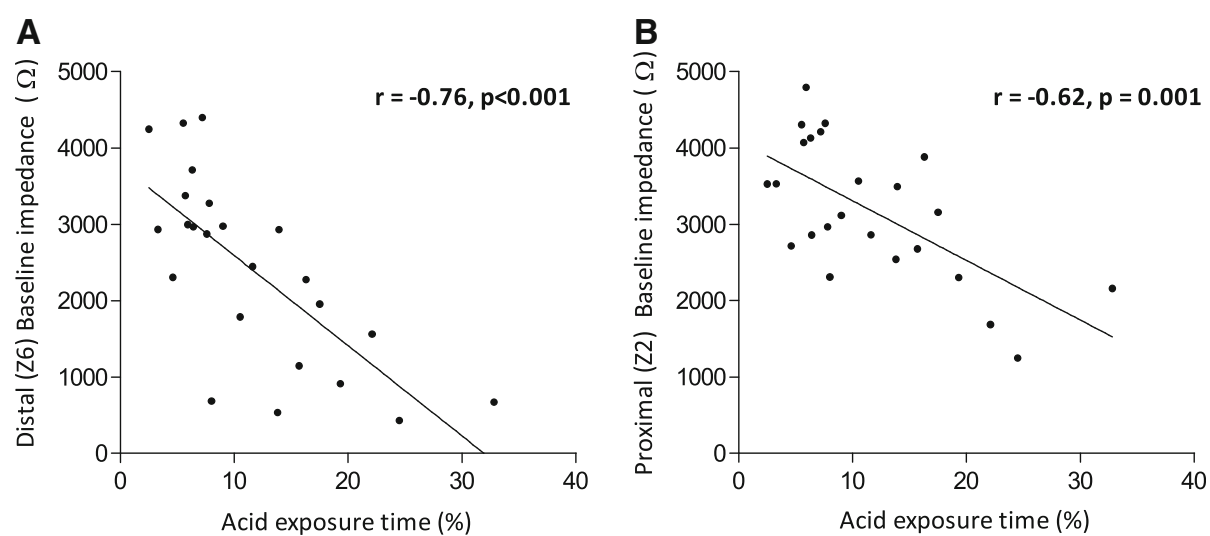
Table 2 Reflux parameters on MII-pH monitoring

\begin{tabular}{lccr}
\hline & Preoperative & Postoperative & $p$ value \\
\hline Acid exposure total $(\%$, IQR) & $8.5(6.0-16.2)$ & $0.8(0.2-2.8)$ & $<0.001$ \\
Upright $(\%$, IQR) & $12.1(4.8-19.2)$ & $1.8(0.5-5.9)$ & 0.001 \\
Supine $(\%$, IQR) & $7.1(0.9-15.3)$ & $0(0-0)$ & $<0.001$ \\
Total GER $(n, \mathrm{IQR})$ & $92(66-139)$ & $14(11-22)$ & $<0.001$ \\
Acid GER $(n, \mathrm{IQR})$ & $61(34-94)$ & $8(1-13)$ & $<0.001$ \\
Non-acid GER $(n, \mathrm{IQR})$ & $23(11-42)$ & $5(3-11)$ & $<0.001$ \\
Proximal reflux $(n, \mathrm{IQR})$ & $36(14-87)$ & $1(0-3)$ & $<0.001$ \\
\hline
\end{tabular}

GER Gastroesophageal reflux, $n$ number of reflux episodes, $I Q R$ interquartile range

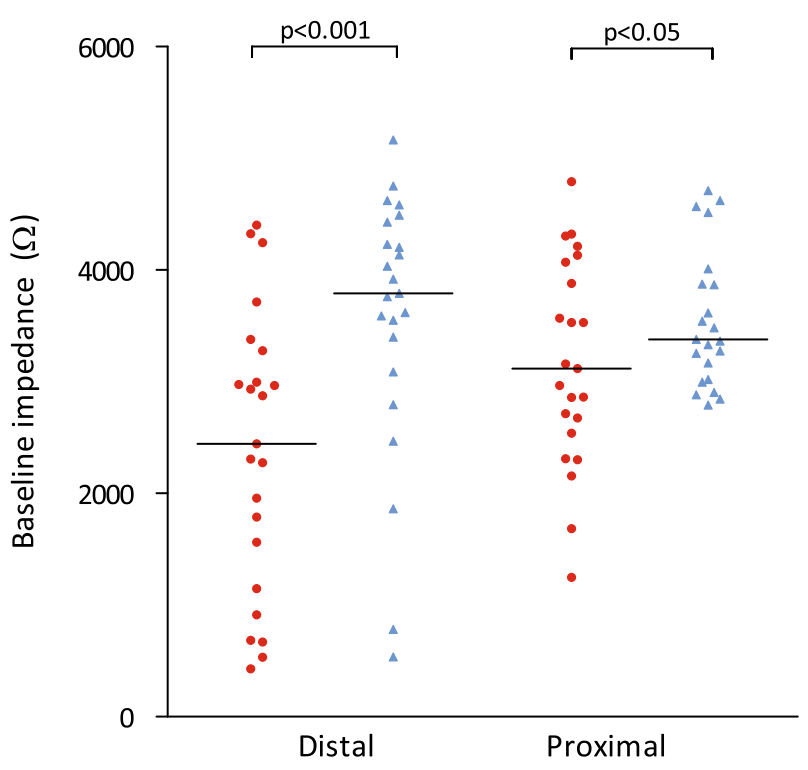

Fig. 2 Baseline impedance in distal (z6) and proximal (z2) esophageal segments before (red) and after (blue) LARS

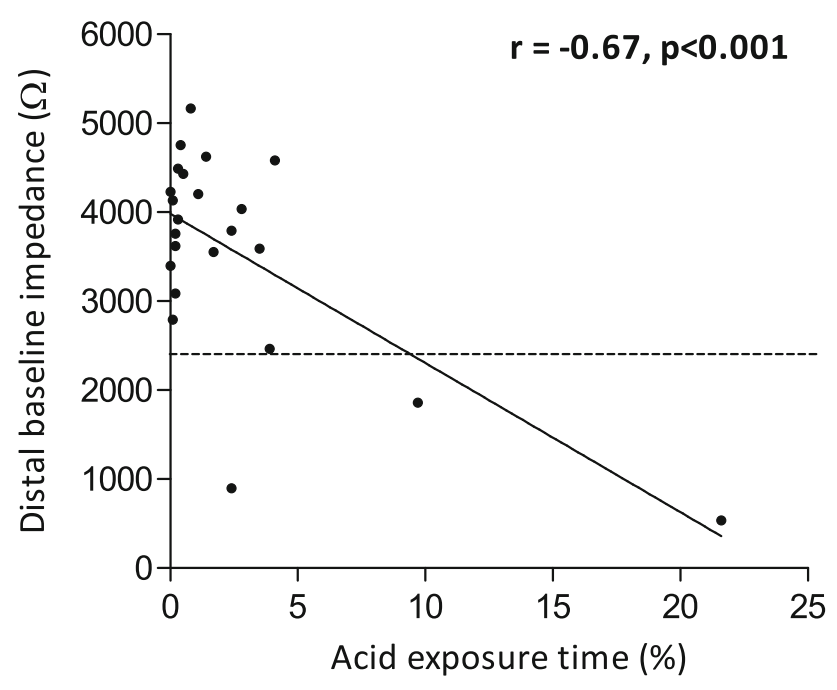

Fig. 3 Correlation between acid exposure time (\%) distal baseline impedance $(\Omega)$ and after LARS. The horizontal dashed line indicates preoperative median baseline impedance
Table 3 Symptom assessment

\begin{tabular}{lcll}
\hline & Preoperative $(n, \%)$ & Postoperative $(n, \%)$ & $p$ value \\
\hline \multicolumn{2}{l}{ Reflux symptoms } & & \\
None & $0(0 \%)$ & $1(65 \%)$ & \\
Mild & $2(9 \%)$ & $5(22 \%)$ & 0.001 \\
Moderate & $6(26 \%)$ & $2(9 \%)$ & \\
Severe & $15(65 \%)$ & $1(4 \%)$ & \\
\hline
\end{tabular}

Age $(\beta$ : $-3.5 \Omega ; 95 \% \mathrm{CI}-114.6 ; 107.7, p=0.95)$ and type of fundoplication $(\beta$ : $-105.1 \Omega ; 95 \%$ CI -1274.7 ; 1064.5, $p=0.95$ ) did not affect the change in baseline impedance after LARS. Also, the change in acid exposure time $(\beta: 50.7 \Omega ; 95 \% \mathrm{CI}-10.3 ; 111.7, p=0.098)$ did not reach statistical significance.

\section{Discussion}

In the present study, we investigated the effect of laparoscopic antireflux surgery (LARS) on baseline impedance as a reflection of mucosal integrity in pediatric patients with gastroesophageal reflux disease (GERD). Our main findings were that LARS resulted in recovery of baseline impedance, that reflux symptoms significantly decreased after LARS, without an association with an increase in baseline impedance and finally that no factors affecting the effect of LARS on baseline impedance could be identified.

LARS aims to prevent reflux events from the stomach thereby protecting the esophageal mucosa from potential stressors in the refluxate. Previous studies have shown a strong inverse correlation between baseline impedance and acid exposure in pediatric GERD patients [9, 12, 22]. Accordingly, we hypothesized that successful elimination of esophageal acid exposure by LARS would increase baseline impedance. Our current study confirms this hypothesis as it demonstrates a significant decrease in acid exposure time and an increase in baseline impedance, which likely reflects the recovery of the esophageal mucosa. Furthermore, postoperative low distal baseline 
impedance was observed in patients with persistent pathologic acid exposure time and in one patient with gastric metaplasia. In the latter patient, this could be due to the intrinsic higher conductive properties of columnar epithelium when compared to esophageal squamous epithelium [23].

Impaired mucosal integrity has been proposed as an important mechanism in symptom generation, as it allows acid reflux to permeate into the deeper layers of the mucosa and activate sensory nerve endings [24]. Previous studies have shown an association between esophageal mucosal integrity and acid sensitivity using a standardized acid perfusion test $[25,27]$. Lower baseline impedance, as a marker of impaired mucosal integrity, has also been shown to correlate with clinical signs of GERD in the pediatric population [12]. Related to these findings, recovery of impaired mucosal integrity could hypothetically result in improvement of reflux symptoms in GERD patients [6]. Laparoscopic antireflux surgery showed good clinical outcome, but an association between GERD symptoms and baseline impedance despite the use of validated questionnaires could not be identified. These results are in accordance with previous studies attempting to identify a similar association after PPI treatment in children or after endoscopic fundoplication in adults [12, 13]. Together, these outcomes suggest that in addition to the mucosal integrity, also content, proximal extent and volume of the refluxate, as well as peripheral and central-mediated sensitivity, affect GERD symptom perception [26, 27].

Baseline impedance may allow identification of patients with impaired mucosal integrity after LARS, as it has been associated with esophagitis and microscopic changes of the mucosa $[6,9,10,28]$. Post-procedural evaluation of GERD is important as patients with persistent GERD may be at risk of developing complications, such as esophagitis, stenosis, Barrett's epithelium and ultimately adenocarcinoma of the esophagus [29]. GERD symptoms may not always be evident, especially in younger children or children with impaired neurodevelopment. Evaluation of mucosal impedance during ambulatory 24-h MII-pH testing may help to detect impaired mucosal integrity. MII-pH analysis is, however, a time-consuming and the procedure itself may be uncomfortable for pediatric patients. Recently, other groups have reported on endoscopy guided single-sensor catheters to measure mucosal impedance during endoscopy [30, 31]. These catheters enable instant evaluation of mucosal integrity during endoscopy instead of the normal 24-h MII-pH monitoring. Unfortunately, in children endoscopy is generally performed under general anesthesia. Because of the impact and risks of the anesthesia and the invasive way endoscopy is performed, this method in its current form seems unsuitable in pediatric patients. Future developments may lead to alternative methods that allow a similar instant evaluation of the esophageal mucosa without using endoscopy [32].

In the proximal esophagus, an inverse correlation between baseline impedance and (distal) acid exposure time was seen, whereas no correlation with the number of proximal reflux episodes was found. As the MII-pH catheter used in this study only had a distal $\mathrm{pH}$ sensor, it is not entirely clear whether proximal baseline impedance is directly affected by acid reflux reaching the proximal esophagus or by indirect mechanisms, activated by distal acid exposure [33]. Previously, Farre et al. [33] showed that distal acid perfusion resulted in changes in the mucosal integrity of the non-exposed proximal esophagus, which is suggestive for an indirect mucosal reaction spreading more proximally than the site of mucosal injury. Further insights in this possible mechanism of impaired mucosal integrity in the proximal esophagus may be of clinical importance as the proximal esophagus is often linked to symptom perception [34-36].

Determinants, such as age, type of fundoplication or change in acid exposure time, influencing the effect of LARS on baseline impedance could not be identified. Infants and young children previously were shown to have a lower baseline impedance when compared to older (pediatric) patients [37]. Age, however, did not influence the effect of LARS on baseline impedance. Until now, studies on efficacy of different types of fundoplication in both the adult and pediatric population were not able to show differences in reflux control [38, 39]. In this study type of fundoplication also was not a significant determinant for the effect of LARS on baseline impedance. The change in acid exposure time after LARS showed a tendency to influence the effect of LARS on baseline impedance and may have been a significant determinant, if more patients had been included.

The main limitation in this current study is the number of patients. A larger number of patients would have allowed us to investigate determinants of interest in a linear regression model with higher statistical power. Furthermore, in the current study endoscopy was not performed per protocol. In 18 out of 25 (72\%) patients, endoscopy was performed before LARS and in only a few of the patients after the procedure. Data to correlate the changes in baseline impedance with endoscopic and/or histological mucosal findings are therefore not available. Pardon et al. showed in adults that baseline impedance is closely correlated to evaluation of mucosal integrity by established ex vivo methodology (Ussing Chamber technique) [10]. Together with previous findings from an experimental model [6], these observations indicate that baseline impedance is a reliable tool for the evaluation of mucosal integrity in vivo.

In conclusion, LARS increased baseline impedance, which is likely to reflect the recovery of mucosal integrity. 
Although both baseline impedance and symptomatic reflux control increased, these two parameters were not mutually associated. Factors influencing the effect of LARS on baseline impedance could not be identified.

Acknowledgments Declaration of personal and funding interests: F.A. Mauritz is supported by a Wilhelmina Children's Hospital Grant and NutsOhra Grant. No. other sources of support were used.

Author contributions FAM and NFR contributed to study design, acquisition of data, analysis and interpretation of data, drafting of the manuscript. EVH and CES helped in acquisition of data and critical revision of the manuscript. PDS, RH, AAM, and JMC contributed to critical revision of the manuscript. DCZ helped in study design, acquisition of data, and critical revision of the manuscript. MHL helped in study design, acquisition of data, analysis and interpretation of data, and drafting and revision of the manuscript.

\section{Compliance with ethical standards}

Disclosures Femke A. Mauritz, Nicolaas F. Rinsma, Ernest L. W. van Heurn, Cornelius E. J. Sloots, Peter D. Siersema, Roderick H. J. Houwen, David C. van der Zee, Ad A. M. Masclee, José M. Conchillo and Maud Y. A. Van Herwaarden-Lindeboom have no conflicts of interest or financial ties to disclose

Open Access This article is distributed under the terms of the Creative Commons Attribution 4.0 International License (http://crea tivecommons.org/licenses/by/4.0/), which permits unrestricted use, distribution, and reproduction in any medium, provided you give appropriate credit to the original author(s) and the source, provide a link to the Creative Commons license, and indicate if changes were made.

\section{References}

1. Skopnik H, Silny J, Heiber O et al (1996) Gastroesophageal reflux in infants: evaluation of a new intraluminal impedance technique. J Pediatr Gastroenterol Nutr 23(5):591-598

2. Sifrim D, Silny J, Holloway RH et al (1999) Patterns of gas and liquid reflux during transient lower oesophageal sphincter relaxation: a study using intraluminal electrical impedance. Gut 44(1):47-54

3. Rosen R, Lord C, Nurko S (2006) The sensitivity of multichannel intraluminal impedance and the $\mathrm{pH}$ probe in the evaluation of gastroesophageal reflux in children. Clin Gastroenterol Hepatol 4(2): 167-172

4. van Wijk MP, Benninga MA, Omari TI (2009) Role of the multichannel intraluminal impedance technique in infants and children. J Pediatr Gastroenterol Nutr 48(1):2-12

5. van Wijk MP, Sifrim D, Rommel N et al (2009) Characterization of intraluminal impedance patterns associated with gas reflux in healthy volunteers. Neurogastroenterol Motil 21(8):825-e55

6. Farre R, Blondeau K, Clement D et al (2011) Evaluation of oesophageal mucosa integrity by the intraluminal impedance technique. Gut 60(7):885-892

7. Shay S (2004) Esophageal impedance monitoring: the ups and downs of a new test. Am J Gastroenterol 99(6):1020-1022

8. Kandulski A, Weigt J, Caro C et al (2015) Esophageal intraluminal baseline impedance differentiates gastroesophageal reflux disease from functional heartburn. Clin Gastroenterol Hepatol 13(6): 1075-1081
9. Borrelli O, Salvatore S, Mancini V et al (2012) Relationship between baseline impedance levels and esophageal mucosal integrity in children with erosive and non-erosive reflux disease. Neurogastroenterol Motil 24(9):828-e394

10. Pardon N, Blondeau K, Vanheel $\mathrm{H}$ et al (2012) In vivo and in vitro evaluation of esophageal mucosal integrity in healthy humans and GERD patients. Gastroenterology 142:S146-S147.

11. Kessing BF, Bredenoord AJ, Weijenborg PW et al (2011) Esophageal acid exposure decreases intraluminal baseline impedance levels. Am J Gastroenterol 106(12):2093-2097

12. Loots CM, Wijnakker R, van Wijk MP et al (2012) Esophageal impedance baselines in infants before and after placebo and proton pump inhibitor therapy. Neurogastroenterol Motil 24(8):758-762 (e351-2)

13. Rinsma NF, Farre R, Bouvy ND et al (2015) The effect of endoscopic fundoplication and proton pump inhibitors on baseline impedance and heartburn severity in GERD patients. Neurogastroenterol Motil 27(2):220-228

14. Fuchs KH, Babic B, Breithaupt W et al (2014) EAES recommendations for the management of gastroesophageal reflux disease. Surg Endosc 28(6):1753-1773

15. Thal AP (1968) A unified approach to surgical problems of the esophagogastric junction. Ann Surg 168(3):542-550

16. Nissen R (1956) A simple operation for control of reflux esophagitis [Eine einfache Operation zur Beeinflussung der Refluxoesophagitis]. Schweiz Med Wochenschr 86(Suppl 20):590-592

17. Mauritz FA, van Herwaarden-Lindeboom MY, Zwaveling S et al (2014) Laparoscopic Thal fundoplication in children: a prospective 10- to 15-year follow-up study. Ann Surg 259(2):388-393

18. Deal L, Gold BD, Gremse DA et al (2005) Age-specific questionnaires distinguish GERD symptom frequency and severity in infants and young children: development and initial validation. J Pediatr Gastroenterol Nutr 41(2):178-185

19. Sifrim D, Holloway R, Silny J et al (2001) Acid, nonacid, and gas reflux in patients with gastroesophageal reflux disease during ambulatory 24-h pH-impedance recordings. Gastroenterol 120(7):1588-1598 (Epub 2001/05/29)

20. Richter JE, Bradley LA, DeMeester TR et al (1992) Normal 24-h ambulatory esophageal $\mathrm{pH}$ values. Influence of study center, $\mathrm{pH}$ electrode, age, and gender. Dig Dis Sci 37(6):849-856

21. Smout AJ, Breedijk M, van der Zouw C et al (1989) Physiological gastroesophageal reflux and esophageal motor activity studied with a new system for 24-h recording and automated analysis. Dig Dis Sci 34(3):372-378

22. Pilic D, Hankel S, Koerner-Rettberg C et al (2013) The role of baseline impedance as a marker of mucosal integrity in children with gastro esophageal reflux disease. Scand J Gastroenterol 48(7):785-793

23. Hemmink GJ, Alvarez Herrero L, Bogte A et al (2013) Esophageal motility and impedance characteristics in patients with Barrett's esophagus before and after radiofrequency ablation. Eur J Gastroenterol Hepatol 25(9):1024-1032

24. Barlow WJ, Orlando RC (2005) The pathogenesis of heartburn in nonerosive reflux disease: a unifying hypothesis. Gastroenterol 128(3):771-778

25. Woodland $\mathrm{P}, \mathrm{Al}$-Zinaty M, Yazaki E, et al (2012) In vivo evaluation of acid-induced changes in oesophageal mucosa integrity and sensitivity in non-erosive reflux disease. Gut 62(9):1256-1261.

26. Bredenoord AJ (2012) Mechanisms of reflux perception in gastroesophageal reflux disease: a review. Am J Gastroenterol 107(1):8-15

27. Weijenborg PW, Smout AJ, Verseijden C et al (2014) Hypersensitivity to acid is associated with impaired esophageal mucosal integrity in patients with gastroesophageal reflux disease 
with and without esophagitis. Am J Physiol Gastrointest Liver Physiol 307(3):G323-G329

28. Salvatore S, Salvatoni A, Ummarino D et al (2016) Low mean impedance in 24-h tracings and esophagitis in children: a strong connection. Dis Esophagus 29(1):10-14

29. Jeurnink SM, van Herwaarden-Lindeboom MY, Siersema PD et al (2011) Barrett's esophagus in children: does it need more attention? Dig Liver Dis 43(9):682-687

30. Ates F, Yuksel ES, Higginbotham $T$ et al (2015) Mucosal impedance discriminates GERD from non-GERD conditions. Gastroenterol 148(2):334-343

31. Weijenborg PW, Rohof WO, Akkermans LM et al (2013) Electrical tissue impedance spectroscopy: a novel device to measure esophageal mucosal integrity changes during endoscopy. Neurogastroenterol Motil 25(7):574-578

32. Fass R (2015) Esophageal mucosal impedance: is it time to forgo prolonged gastroesophageal reflux recordings? Gastroenterol 148(2):282-285

33. Farre R, Fornari F, Blondeau K et al (2010) Acid and weakly acidic solutions impair mucosal integrity of distal exposed and proximal non-exposed human oesophagus. Gut 59(2):164-169

34. Bredenoord AJ, Weusten BL, Timmer R et al (2006) Characteristics of gastroesophageal reflux in symptomatic patients with and without excessive esophageal acid exposure. Am J Gastroenterolog 101(11):2470-2475

35. Cicala M, Emerenziani S, Caviglia R et al (2003) Intra-oesophageal distribution and perception of acid reflux in patients with non-erosive gastro-oesophageal reflux disease. Aliment Pharmacol Ther 18(6):605-613

36. Woodland P, Aktar R, Mthunzi E et al (2015) Distinct afferent innervation patterns within the human proximal and distal esophageal mucosa. Am J Physiol Gastrointest Liver Physiol 308(6):G525-G531

37. Salvatore S, Salvatoni A, Van Berkel M et al (2013) Esophageal impedance baseline is age dependent. J Pediatr Gastroenterol Nutr 57(4):506-513

38. Broeders JA, Mauritz FA, Ahmed Ali U et al (2010) Systematic review and meta-analysis of laparoscopic Nissen (posterior total) versus Toupet (posterior partial) fundoplication for gastro-oesophageal reflux disease. Br J Surg 97(9):1318-1330

39. Mauritz FA, Blomberg BA, Stellato RK et al (2013) Complete versus partial fundoplication in children with gastroesophageal reflux disease: results of a systematic review and meta-analysis. J Gastrointest Surg 17(10):1883-1892 